Planetary Systems in the Universe - Observation, Formation and Evolution

Proceedings IAU Symposium No. 202, (c)2004 IAU

Alan Penny, Pawel Artymowicz, Anne-Marie Lagrange, 8 Sara Russell, eds.

\title{
The ESO radial velocity program. Status and results
}

\author{
Martin Kürster ${ }^{1}$, Michael Endl ${ }^{2}$, Sebastian Els ${ }^{3}$, Artie P. Hatzes ${ }^{4}$, \\ Willam D. Cochran ${ }^{5}$, Frédéric Rouesnel ${ }^{6}$, Konrad Dennerl $^{7}$ and \\ Stefan Döbereiner ${ }^{7}$ \\ ${ }^{1}$ European Southern Observatory, Casilla 19001, Santiago, Chile \\ ${ }^{2}$ Institut für Astronomie, Universität Wien, Türkenschanzstr. 17, \\ A-1180 Wien, Austria \\ ${ }^{3}$ Institut für Theoretische Astrophysik, Universität Heidelberg, \\ Tiergartenstr. 15, D-69121 Heidelberg, Germany \\ ${ }^{4}$ Thüringer Landessternwarte Tautenburg, Sternwarte 5, D-07778 \\ Tautenburg, Germany \\ ${ }^{5}$ McDonald Observatory, The University of Texas at Austin, Austin, \\ $T X$ 78718-1083, USA \\ ${ }^{6}$ Université de Paris-Sud, Bâtiment 470, F-91405 Orsay Cedex, France \\ ${ }^{7}$ Max-Planck-Institut für extraterrestrische Physik, Giessenbachstr., \\ D-85740 Garching, Germany
}

\begin{abstract}
We present results from our precision radial velocity (RV) program at ESO La Silla. The achievable RV precision after the upgrade of the CES spectrograph is demonstrated. We apply the method of pooled variance diagrams to compare our short-term with our long-term precision. For the active planet-hosting star $\iota$ Hor we determine the influence of stellar activity and the rotation period, and address the question of a second long-period planet. For the RV signal in $\epsilon$ Eri we demonstrate the distinctness of the time scales present in the RV and CaII data, providing further support for the planetary interpretation in this active star.
\end{abstract}

\section{Instrumental developments in the ESO CES planet survey}

Our program in search for extrasolar planets in the southern hemisphere was begun in Nov. 1992 at ESO La Silla using the 1.4m CAT telescope and the CES spectrograph which yielded during the first $51 / 2$ years a spectrum length of $48.5 \AA$ (centered on $5389 \AA$ ) and a resolving power of $R=100,000$. To achive high measurement precision for precise radial velocities (RV) we self-calibrate the CES with a sealed and temperature controlled iodine gas absorption cell. Our data modelling approach involves the detailed reconstruction of the instrumental profile and is outlined in Endl, Kürster \& Els (2000).

The self-calibration approach made it possible to continue our survey during a sequence of instrument upgrades that have occurred since April 1998. First, the new Very Long Camera increased the resolving power to about $R=200,000$ but at the same time reduced our bandwidth to only $18.2 \AA$. In Oct. 1998 the CAT telescope was decommissioned and the CES fibre-coupled to the ESO $3.6 \mathrm{~m}$ telescope with image slicers securing a high instrumental efficiency as well 
as a high resolving power of $R=230,000$. In the last stage a longer CCD was installed in Sept. 1999 with which we now achieve a spectral bandwidth of $36.5 \AA$. This final configuration makes it possible to obtain high-quality template spectra (without the iodine cell) of our program stars that can be used in the modelling process (for details see Endl, Kürster \& Els 2000) for the whole data set (back to 1992) with best results.

Fig. 1 shows the RV data of the K4V star GJ 570A = HR 5568 during the various phases of the instrument upgrade, modelled using stellar templates from the most recent phase. The best precision, $3.2 \mathrm{~ms}^{-1} \mathrm{rms}$, is obtained for the data from the most recent configuration (open triangles); note that this is a short-term precision, from which we expect a long-term precision of about $5 \mathrm{~ms}^{-1}$ which needs to be verified. Offsets of a few $10 \mathrm{~ms}^{-1}$ are seen for the data with half the spectral bandwidth (open squares and open circles), obviously as a result of the fact that the RVs could only be inferred from part of the spectral lines. However, no offset is seen with respect to the earliest configuration (filled diamonds) since of the spectra obtained during this phase only the bandwidth coinciding with the most recent configuration was used.

\section{The Pooled Variance Analysis Tool}

We have started to apply the tool of Pooled Variance Diagrams (PVD) to our RV data. This method was first introduced by Dobson et al. (1990) to study time series of the CaII emission strength of active late-type stars, but the method is applicable to any kind of time series data. Dobson et al. (1990) and Donahue \& Dobson (1996) demonstrated the capability of this technique to detect time scales pertaining to instrumental effects or to stellar activity, e.g.: major changes in the instrument stability (i.e. setup changes), modulation of visibility and RV of active regions with stellar rotation, the typical time scale of active region reconfigurations, and the stellar activity cycle.

These processes show characteristic time scales, but they are not strictly periodic. Instrumental stability degrades whenever there is a major change of instrument setup. Rotational modulation of active regions (in late-type stars) has a fundamental period which is the stellar rotation period, however, reconfigurations of active regions lead to signals that change phase, amplitude, and shape thereby preventing their discovery with Fourier or periodogram techniques. The time scale of active region reconfiguration itself usually does not display periodicities. Stellar long-term activity behaviour is cyclic only for stars with low activity levels (like the Sun), but exhibits more complexity in active stars.

Analysis via PVDs examines the total variance in a time series of measurements as a function of the length of selected time intervals within the series. We subdivide the total time baseline $T$ of our data set into intervals $T, T / 2, T / 3, \ldots$, evaluate the temporal distribution of points within each of these windows in order to determine the effective time they span, calculate the variance for each window, group windows of similar length together, and calculate the average variance for the resulting time scales (window groups).

Every effect that occurs with a characteristic time scale contributes little to the total scatter at much shorter time intervals, contributes more and more to the scatter at time intervals whose length approaches the characteristic time 


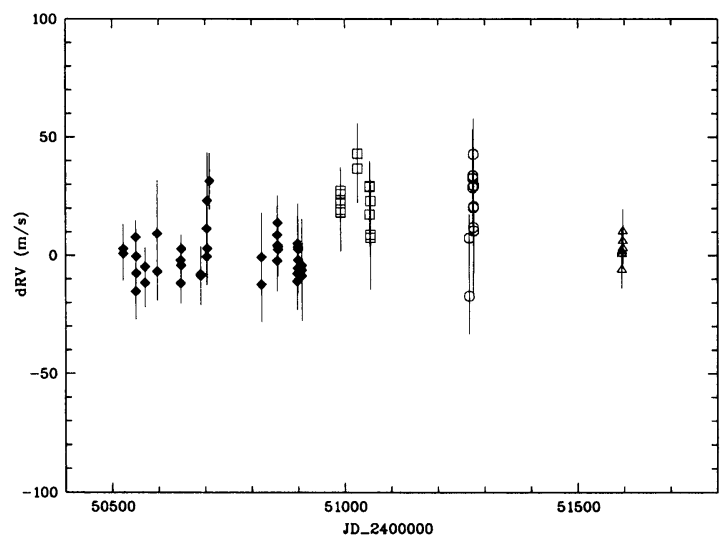

Figure 1. RV data of the K4V star GJ 570A (=HR 5568) as observed during the various phases of the CES upgrade. Filled diamonds: CAT telescope, $R=100,000$, bandwidth $48.5 \AA$; open squares: CAT telescope, $R=200,000$, bandwidth $18.2 \AA$; open circles: $3.6 \mathrm{~m}$ telescope, $R=230,000$, bandwidth $18.2 \AA$; open triangles: $3.6 \mathrm{~m}$ telescope, $R=230,000$, bandwidth $36.5 \AA$.

scale of the effect, but does not increase the scatter any further at times much longer than its characteristic time scale. Therefore, we are looking in the PVD for features representing an increase in variance followed by a plateau which occurs near the characteristic time scale of the underlying effect.

\section{Instrumental short-term vs. long-term precision}

Fig. 2 shows the time series of our data from the first survey configuration for the G8V star $\tau$ Cet. The PVD derived from these data is presented in Fig. 3. At the level of our measurement precision this star does not exhibit any RV variability of its own. A plateau-like structure is seen in the PVD which indicates a variability time scale of about 68 days which is comparable to a two-month data spacing occurring frequently in our data set (but see next two paragraphs). We interpret this effect as the difference between short-term (few nights during which the instrumental setup is kept fixed) and long-term precision (i.e. after instrumental setup changes). In this early survey configuration (1992-1998) our long-term precision (11.3 $\mathrm{ms}^{-1}$ for $\tau$ Cet) is about a factor 1.5 worse than our short-term precision ( $7.5 \mathrm{~ms}^{-1}$ for $\tau$ Cet) indicating the final limitations we face with our technique of instrumental profile modelling.

We model this behaviour by chosing a combination of two types model functions: a) "white noise", i.e. a constant rms-component independent from the underlying time scale; and b) the average PVD of sine-waves with a given period and (semi-)amplitude, where the average is performed as an ensembleaverage with respect to the phase of the sine-waves. The white noise component can be interpreted as any kind of instrumental precision limit, whereas the phaseaveraged PVD of sine waves is taken as representative of any kind of variability occurring with a characteristic time scale. Even in the case of strictly periodic 


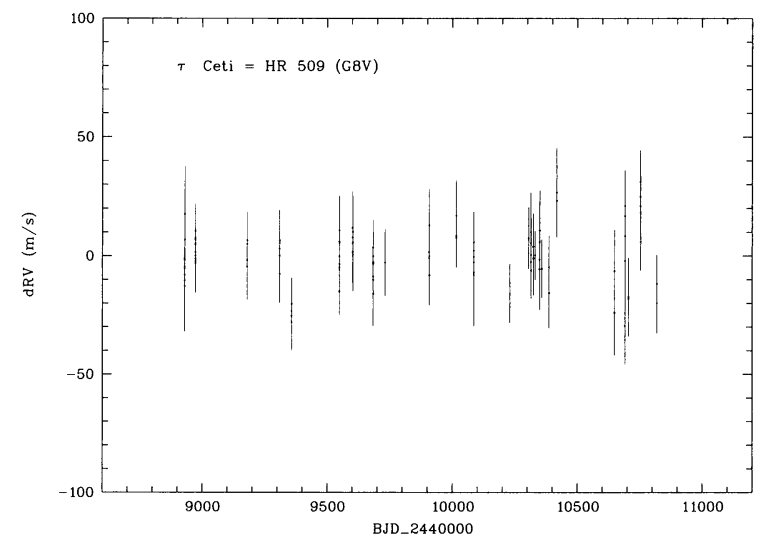

Figure 2. RV time series for $\tau$ Ceti. All data were obtained with the first instrument configuration.

signals phase averaging occur naturally in many observational circumstances due to irregular sampling, except if a studied time scale is a multiple of the underlying period.

The dashed line in Fig. 3 shows such a model comprised of white noise with an rms of $7.5 \mathrm{~ms}^{-1}$ plus a model function with a time scale of $68 \mathrm{~d}$ and a (semi-)amplitude of $12 \mathrm{~ms}^{-1}$. For this RV constant-star the model should be taken as illustrative only; the true behaviour probably does not have such a clear-cut time scale, but is more likely a superposition of a range of time scales from weeks to several months as indicated by the fact that the rise in the PVD points is less steep than in the model. The value of 68 day determined for the single time scale model is probably somewhere near the median value of this time-scale range.

\section{Stellar activity effects in $\iota$ Hor}

Fig. 4 shows the PVD of our RV data for $\iota$ Hor, an active G0V star around which we have found an extrasolar planet with a period of $320 \mathrm{~d}$ (see Kürster et al. 2000 for the time series of the RV data). To represent this diagram we applied a complex model shown as the dashed line in the figure. It consists of a white noise component with an rms of $14.6 \mathrm{~ms}^{-1}$ plus four variable components with time scales of $6.5 \mathrm{~d}, 90 \mathrm{~d}, 320 \mathrm{~d}$, and $2000 \mathrm{~d}$, and (semi-)amplitudes of $18.0 \mathrm{~ms}^{-1}, 31.0 \mathrm{~ms}^{-1}, 60.0 \mathrm{~ms}^{-1}$, and $35.8 \mathrm{~ms}^{-1}$, respectively.

The third component is clearly attributable to the signal of the planet for which we had found a slightly eccentric orbit with $P=320.1 \pm 2.1 \mathrm{~d}, K=$ $67.0 \pm 5.1 \mathrm{~ms}^{-1}$, and $e=0.161 \pm 0.069$ (Kürster et al. 2000).

We interpret the first component as due to rotational modulation of active regions in this active star. This can be seen as follows. From our paper (Kürster et al. 2000) we take the projected stellar rotation velocity $v \sin i=5.5 \pm 0.5 \mathrm{kms}^{-1}$ and the stellar radius of $R=1.097 \pm 0.019 \mathrm{R}_{\odot}$. We combine these values with 


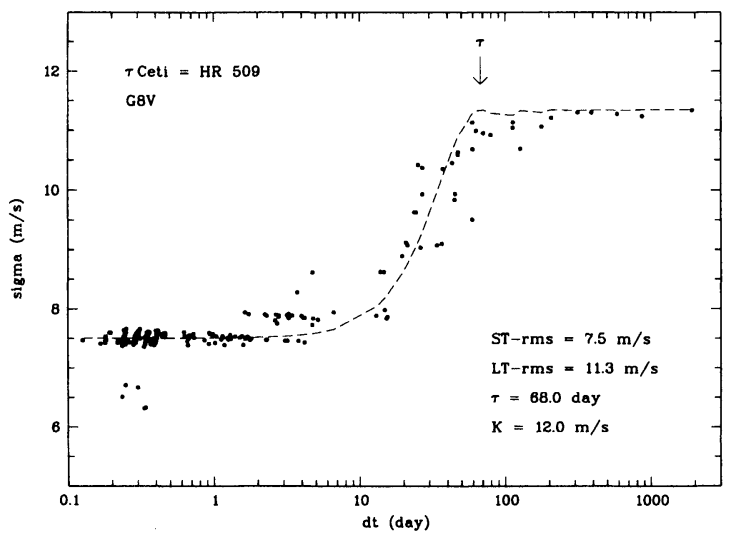

Figure 3. PVD for the RV data of $\tau$ Ceti shown in Fig. 2. The average $\mathrm{rms}$ is plotted as a function of the pertaining time scale. The dashed line is a theoretical curve as described in the text; it corresponds to a variation with a (semi-)amplitude of $K=12.0 \mathrm{~ms}^{-1}$ with a characteristics time scale $\tau=68.0 \mathrm{~d}$.

our recent discovery of a dust disk around $\iota$ Hor (Els et al. 2000) which has an inclination of $i=41.5^{\circ} \pm 3.5^{\circ}$. We furthermore assume that the stellar inclination is the same as the disk inclination and can thus determine the stellar rotation period as $P_{\text {rot }}=6.69 \pm 0.77 \mathrm{~d}$, in agreement with the time scale of our first model component in the PVD.

The second component does not display a clear plateau, but rather sits in the wing of the rise to the plateau corresponding to the third component. The origin of the component is not absolutely clear, but it is required in the model in order to represent well the PVD at time scales of a few months. With a characteristic time scale of $90 \mathrm{~d}$ we suspect its origin to be partially due to the change from short-term to long-term precision (that we have illustrated in the RV constant star $\tau$ Cet; see Sect. 3 and Fig. 3) and also related to the time scale of the dominant active region reconfiguration events.

Component four is required to model the PVD at the longest time scales, in particular time scales longer than the orbital period of the planet as the total scatter of the data is clearly rising at longer time scales, an effect that can also be appreciated by inspecting the RV residuals from the fit with the planet orbit (Fig. 2b in Kürster et al. 2000). We have chosen a time scale of $2000 \mathrm{~d}$ for component four, but any value between $600 \mathrm{~d}$ and a few thousand days could be used for a satisfactory model. Two possibilities arise for the interpretation of this longest time scale. First, it could be related to the stellar activity cycle or be part of the multi-periodic long-term behavour of active stars. Second, it could indicate a second planet in a long-period orbit around $\iota$ Hor, a possibility which we are currently following up. 


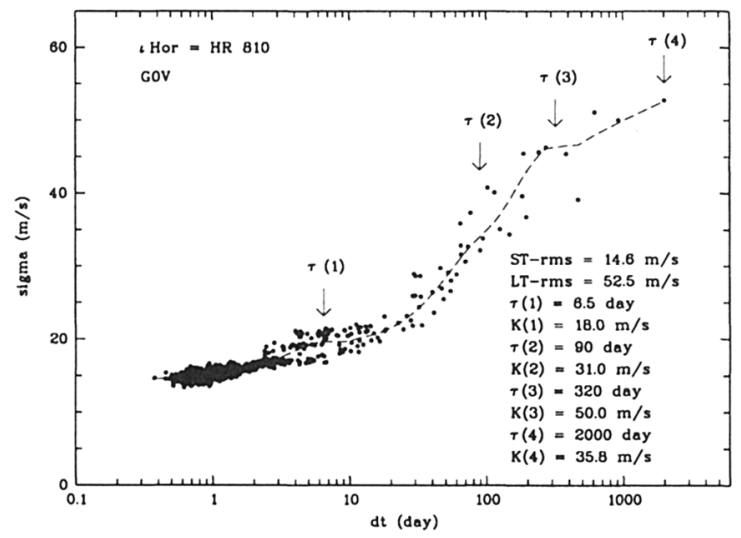

Figure 4. PVD for $\iota$ Horologii. The dashed line corresponds to a model with white noise of $14.6 \mathrm{~ms}^{-1} \mathrm{rms}$ plus four variability components with characteristic time scales $\tau$ and (semi-)amplitudes $K$ as labelled in the figure (see also text).

\section{Stellar activity effects in $\epsilon$ Eri}

The recent discovery of a long-period $(P=6.9 \mathrm{yr})$ planet around the active $\mathrm{K} 2 \mathrm{~V}$ star $\epsilon$ Eri (Hatzes et al. 2000) was based on combined RV data from four different teams including our CES data. The total time baseline of the combined data set spans 19 yr. The RV time series can be found in Hatzes et al. (2000).

Fig. 5 shows the PVD based on the total (combined) RV data set. We model it with a white noise component ( $\mathrm{rms}$ of $15.3 \mathrm{~ms}^{-1}$ ) plus two variable components with time scales of $40.0 \mathrm{~d}$ and $6.9 \mathrm{yr}$. While the first component may be a combination of the effects of activity (rotational modulation) and the transition from short-term to long-term precision, the second component can be attributed to the planet.

In order to rule out an interpretation of the long-period RV signal as due to an activity cycle in this active star Hatzes et al. (2000) used periodogram techniques to study periodicities present in CaII $\mathrm{H}+\mathrm{K}$ S-index data taken during the time interval the RV data were taken (see e.g. Baliunas et al. 1995 for the CaII survey). They found periodicities near $20 \mathrm{yr}, 3 \mathrm{yr}$, and (weaker) $3.8 \mathrm{yr}$. Since none of these coincided with the period of the planet they concluded that the $6.9 \mathrm{yr}$ signal seen in the RV data is not attributable to stellar activity.

Fig. 6 shows the PVD of the CaII H+K S-index data modelled with a white noise component plus three variable components at time scales of $40 \mathrm{~d}, 3 \mathrm{yr}$ and $20 \mathrm{yr}$. The $40 \mathrm{~d}$ component is reminiscent of the first component in the RV data, however, it appears that a continuous range of time scales with values of about $1-10$ weeks might give an even better representation of the short-term behaviour of the PVD of the CaII data that does not display a clear-cut plateau in this temporal regime. The second and third time scales were chosen to match the two strongest periodogram peaks at $20 \mathrm{yr}$ and $3 \mathrm{yr}$ and provide a good representation of the PVD at longer time scales. Especially, the 3 yr variability 


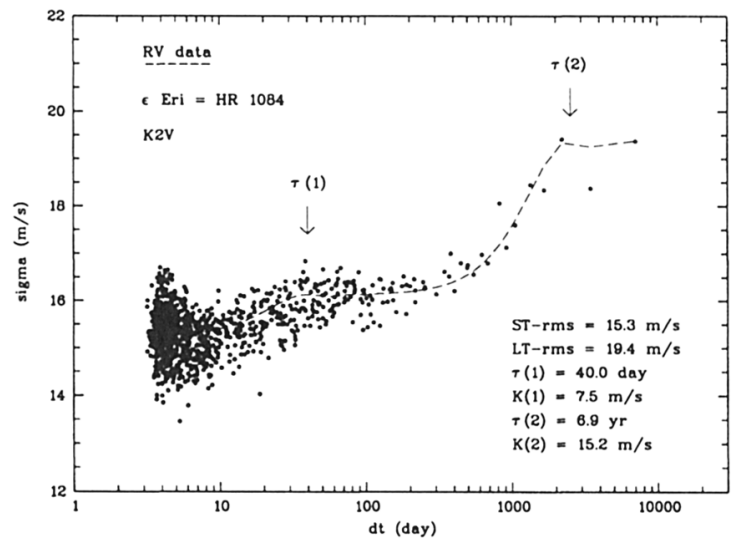

Figure 5. PVD for of $\epsilon$ Eridani based on 19 years of combined RV data from four different groups (see Hatzes et al. 2000). The dashed line corresponds to a model with white noise of $15.3 \mathrm{~ms}^{-1} \mathrm{rms}$ plus two variability components as labelled in the figure.

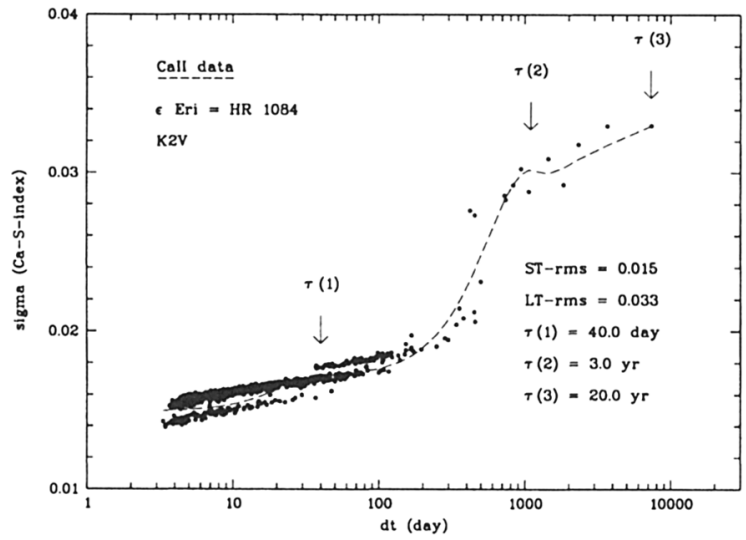

Figure 6. PVD for the CaII data of $\epsilon$ Eridani taken over the same time interval as the RV data that underly Fig. 5. The dashed line corresponds to a model with white noise of 0.015 CaII-index units rms plus three variability components as labelled in the figure. 
is required in order to provide the correct representation of the steepest rise in the PVD. It seems possible still that the $20 \mathrm{yr}$ variability could be replaced by models with somewhat shorter time scales, down to $10 \mathrm{yr}$. However, we prefer the $20 \mathrm{yr}$ time scale as it corresponds to the dominant and highly significant peak in the peridogram and is therefore the best established time scale of all.

We conclude that the PVDs for the RV and CaII data of $\epsilon$ Eri are distinct at time scales longer than about $1 \mathrm{yr}$ which further supports the hypothesis that the $6.9 \mathrm{yr}$ RV signal is not due to stellar activity.

Acknowledgments. We thank the ESO OPC for generous allocation of observing time to this project. M. Endl acknowledges support by the Austrian Fond zur Förderung der wissenschaftlichen Forschung Nr. S7302. W.D. Cochran and A.P. Hatzes acknowledge support by NSF Grant AST-9808980 and NASA Grant NAG5-9227.

\section{References}

Baliunas, S.L., Donahue, R.A., Soon, W.H., Horne, J.H., et al. 1995, ApJ, 438, 269.

Dobson, A.K., Donahue, R.A., Radick, R.R., \& Kadlec, K.L. 1990, Cool Stars, Stellar Systems, and the Sun, 6th Cambridge Workshop, G. Wallerstein (ed.), ASP Conf. Ser. 9, 132

Donahue, R.A., \& Dobson, A.K. 1996, Cool Stars, Stellar Systems, and the Sun, 9th Cambridge Workshop, R. Pallavicini, A.K. Dupree (eds.), ASP Conf. Ser. 109, 599

Els, S., Pantin, E., Marchis, F., Endl M., Kürster M., \& Sterzik M. 2000, Astronomische Gesellschaft, Last Minute Note; and ESO Press Photo 27/00, http://www.eso.org/outreach/press-rel/pr-2000/phot-27-00.html

Endl M., Kürster M., \& Els. S. 2000, A\&A, 362, 585

Endl M., Kürster M., Els S., Hatzes A.P., Cochran W.D., Dennerl K., \& Döbereiner S. 2000, Proc. IAU Symp. 202, Planetary Systems in the Universe: Observation, Formation and Evolution, A. Penny, P. Artymowicz, A.-M. Lagrange, S. Russell (eds.), A.S.P. Conf. Ser. (this volume)

Hatzes A.P., Cochran W.D., McArthur B., Baliunas S.L., Walker G.A.H., Campbell B., Irwin A.W., Yang S., Kürster M., Endl M., Els S., Butler R.P., \& Marcy G.W. 2000, ApJ Letters, in press

Kürster M., Endl M., Els S., Hatzes A.P., Cochran W.D., Döbereiner S., \& Dennerl K. 2000, A\&A, 353 Letter 33 